\title{
Study of Sperm Apoptosis and Seminal Oxidant Capacity in Infertile Patients with Genital Tract Infections Evaluation of the Efficacy of Levofloxacin in Two Therapeutic Protocols
}

\author{
Terradas Claudio, Rodriguez Peña Marcelo, Curi Susana, Grasso Ernesto, Nagelberg Alberto \\ Male Infertility Program, Argentine Society of Urology, Buenos Aires, Argentina \\ Email: cterradas@intramed.net.ar
}

Received October 30, 2011; revised January 31, 2012; accepted February 29, 2012

\begin{abstract}
Introduction: Antibiotics are the principal treatment of seminal tract infections Our objective is to determine the efficacy of levofloxacin in the eradication of genital tract patogens in infertile patients comparing 28 with 56 days of treatment and to elucidate different seminal parameters availables as markers of antibiotic efficacy in seminal improvement. Materials and Methods: We studied prospectively 50 males patients with seminal tract infections. All patients were treated with levofloxacin $500 \mathrm{mg}$ orally along 28 or 56 days. Two seminal analysis were performed before and after treat- ment an seminal parameters included TUNEL and nitric oxide levels in seminal plasma were measured. Results: We observed significative differences between both diagrams of treatment. Sperm count and motility increased significa- tively after treatment. But, sperm morphology do not improve after antibiotic. Necrospermy index but not leucocitospermy had been reduced after levofloxacin. Nitric oxide levels have a direct correlation with sperm count but inverse with motility. The rate of apoptosis determined by TUNEL technique in the study population was $30.46 \%$. There was no correlation between apoptosis parameters and necrozoospermy and there was no correlation between sperm cell apoptosis and NO levels. Conclutions: Bacteriologic cure in seminal infection is higher with a scheme of 56 days than 28 days. Treatment with levofloxacin improves seminal parameters in infertile patients with spermatic tract infection. Nitric oxide but not TUNEL is a good predictive factor of antibiotic efficacy.
\end{abstract}

Keywords: Seminal Tract Infections; Sperm DNA Fragmentation; Nitric Oxide; Levofloxacine

\section{Introduction}

Antibiotics are the main treatment of prostatitis and seminal gland infections. However there is no uniform approach regarding the duration of this treatment by taking into account clinical evidence of initial cure rates, relapse, and bacterial persistence. The indication of an antibiotic may vary by physicians as well as the duration of treatment is necessary to consider properly the extent of antibacterial spectrum, resistance rates and the time required to achieve bactericidal levels in the prostate tissue.

Among the antibiotics that penetrate the prostate half, fluoroquinolones can achieve a concentration in the alkaline medium several times the seminal plasma, which is formed by a group of proven efficacy [1].

In a previous multicenter study it was established that one month of treatment the clinical efficacy rate was $75 \%$ for levofloxacin and $72.8 \%$ for ciprofloxacin, with similar relapse rates for both treatments [2]. It remains to assess whether longer treatment time can get better results.
Several authors have attempted to determine predictors of bacteriological cure regardless of the sperm cultures but there are no reliable markers for it [3]. It would therefore be important to investigate other biochemical markers of seminal tract inflammation as nitric oxide (NO) which would be useful to determine cure or possibility of relapse. Likewise, reactive oxygen species (ROS) may be increased in the seminal plasma of these patients would be useful markers of the efficacy of antibiotic treatment. This has been demonstrated for other diseases such as varicocele $[4,5]$.

The objectives of this work is: 1) Study the efficacy and safety of levofloxacin in eradicating pathogens in the genital tract in infertile patients, 2) Determine the percentage of apoptotic sperm in the semen of men with seminal infection, its correlation with the different parameters of semen and its changes after treatment with levofloxacin in two regimes of different duration and 3) Determine the levels of NO in the semen of these patients before and after antibiotic treatment and evaluating 
the use of these marker as predictor of effective antibiotic therapy. In the present study prospectively studied 50 men with seminal infection who consulted for infertility, aged between 28 and 50. This study excluded all patients with the following conditions: Azoospermy, chronic renal failure, allergy to quinolones, uncompensated diabetes mellitus and previous treatment with antibiotics or antioxidant therapies.

\section{Materials and Methods}

The study population consisted of 50 infertile men with seminal tract infections attending the Centers of Reproductive Medicine at 3 hospitals.

Permission to conduct this study was obtained from the hospital's institutional review board and all subjects gave their consent to participate.

\subsection{Semen Analysis}

For each patient we performed two spermograms before and after antibiotic treatment. Samples were collected by masturbation into a sterile container after 48 to 72 hours of sexual abstinence. They were incubated in culture oven at $37^{\circ} \mathrm{C}$ for 30 minutes to complete seminal liquefaction. Then we evaluated the sperm characteristics (concentration, motility and morphology), sperm morphology was evaluated following two criteria (WHO and Kruger) $[6,7]$.

\subsection{Assessment of Sperm DNA Fragmentation}

The study of DNA fragmentation in ejaculated sperm smear was made by marking technique in situ terminal deoxynucleotidyl transferase - deoxyuridine triphosphate (TUNEL). For this technique, aliquots of semen ejaculated submitted to the laboratory are centrifuged to remove seminal plasma at $400 \mathrm{~g}$ for 10 minutes and then the sperm pellet are spread on slides and fixed in cold acetone or ethanol as appropriate. Smears are incubated with the enzyme, which binds digoxigenin ddUTP associated with the extreme $\mathrm{OH} 3$ 'free of fragmented DNA. To identify nuclei that incorporated digoxigenin-ddUTP be used a second antibody conjugated with fluorescein or rhodamine (Cell Death Detection Kit, Roche, Monza, Italy). The sperm DNA fragmentation were identified by fluorescence microscopy and determined the percentage of TUNEL + spermatozoa.

Negative controls were performed following the steps of the technique but without the addition of Tdt enzyme $[8,9]$.

\subsection{Determination of the Concentration of NO}

As an index of NO production in the spermatic tract was determined nitrite concentration in seminal plasma of all patients and normal controls using the technique of Griess [10]. Briefly, aliquots of $100 \mu \mathrm{L}$ of the incubation media were reacted with $50 \mu \mathrm{L}$ of $1 \%$ sulfanilic acid and then with $50 \mu \mathrm{L}$ hydrochloride $\mathrm{N}$-(1-naphthyl) etildiamina (NEDA) to $0.1 \%$. Incubations were performed in triplicate in 96 holes and the optical density was read after 10 minutes of addition of reagents in a spectrophotometer plate in a plate length of $600 \mathrm{~nm}$. The concentration of nitrite (M) was determined using sodium nitrite as standard.

\subsection{Treatment}

\section{Randomized}

Group a: Levofloxacin $500 \mathrm{mg}$ for 28 days - 25 patients; Group b: Levofloxacin $500 \mathrm{mg}$ for 56 days - 25 patients.

Sexual partners also received antibiotic therapy with levofloxacin $500 \mathrm{mg}$ for 14 days.

\subsection{Statistical Analysis}

We applied nonparametric or parametric test to analyze all the variables as appropriate using a computer program (Instat Graphpad-, USA). We considered significative a $\mathrm{p}$ value $<0.05$.

\section{Results}

There are significant differences between the two treatment regimens (cure rate of $84.7 \%$ for the scheme 28 days and $91.7 \%$ for the scheme 56 days, $\mathrm{p}<0.005$ ). The sperm count improved significantly after antibiotic treatment (pretreatment: $17.2 \pm 8.83$ vs. postreatment: $(35.3 \pm$ $\left.18.32) \times 10^{6} / \mathrm{ml}, \mathrm{p}=0.043\right)$ (Figure 1). Similarly translational sperm motility $(A+B)$ improved significantly after

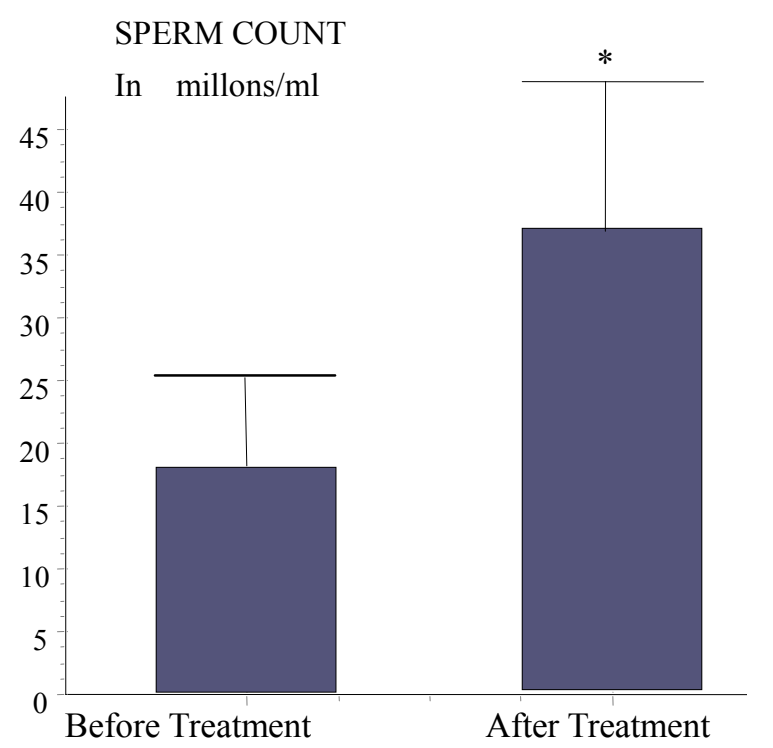

Figure 1. Sperm cell count before and after antibiotic treatment. 
antibiotic treatment (before treatment: $29.71 \pm 30.10$ vs. after treatment: $60.5 \% \pm 17.76 \%, \mathrm{p}=0.029)$ (Figure 2). By contrast, sperm morphology did not differ significantly after treatment (before treatment: $5.16 \% \pm 6.99$ vs. after treatment: $7.16 \% \pm 6.99 \%$ of normal forms according to OMS and Kruger criteria, $\mathrm{p}=0.63$ ). Necrozoospermy index (percentage of dead sperm) decreased significantly after treatment with levofloxacin (before treatment: $61.5 \pm 34.57$ vs. after treatment: $16.33 \% \pm 11.29 \%$, $\mathrm{p}=0.05$ ) (Figure 3). However leukocytospermy parameters did not change significantly (before treatment: $1.50 \pm 1.32$ vs. after treatment: $(1.76 \pm 1.52) \times 10^{6} / \mathrm{ml}, \mathrm{p}$ $=0.78)$.

In this group of patients we observed a direct correlation between NO concentration and sperm count before starting antibiotic treatment (Figure 4) as well as an inverse correlation between $\mathrm{NO}$ concentration and sperm motility $\mathrm{A}+\mathrm{B}$.

We observed a direct correlation between the concentration of NO and necrozoospermy parameters (Figure 5).

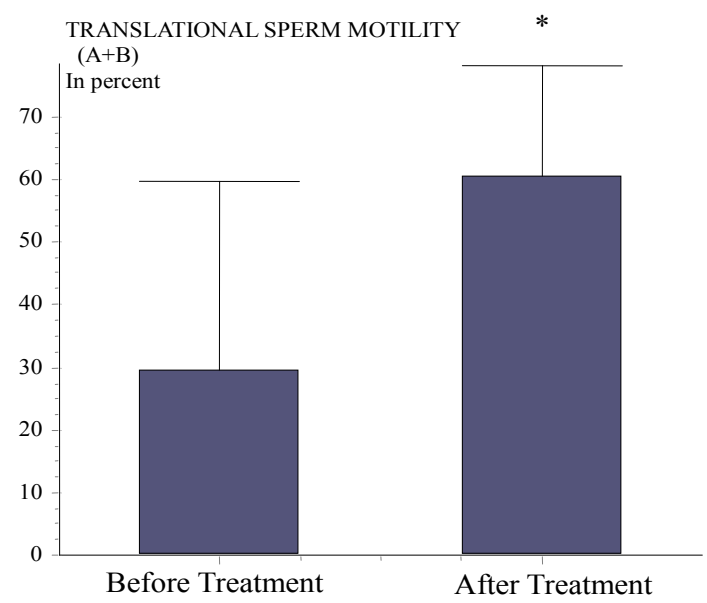

Figure 2. Translational sperm cell motility before and after treatment.

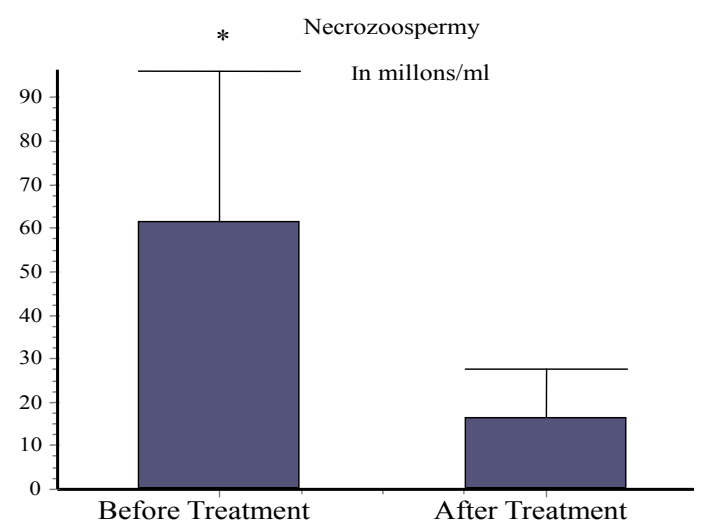

Figure 3. Necrozoospermy before and after treatment with levofloxacine.

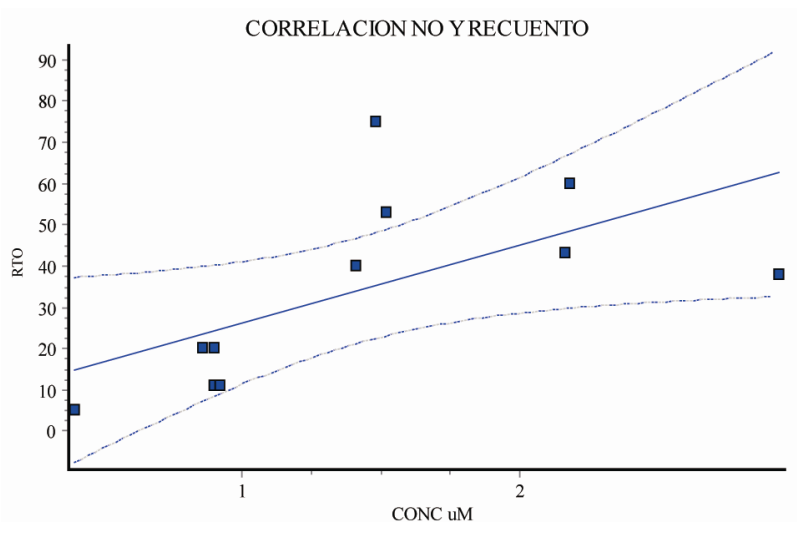

Figure 4. Correlation between seminal NO levels and sperm cell count before and after treatment.

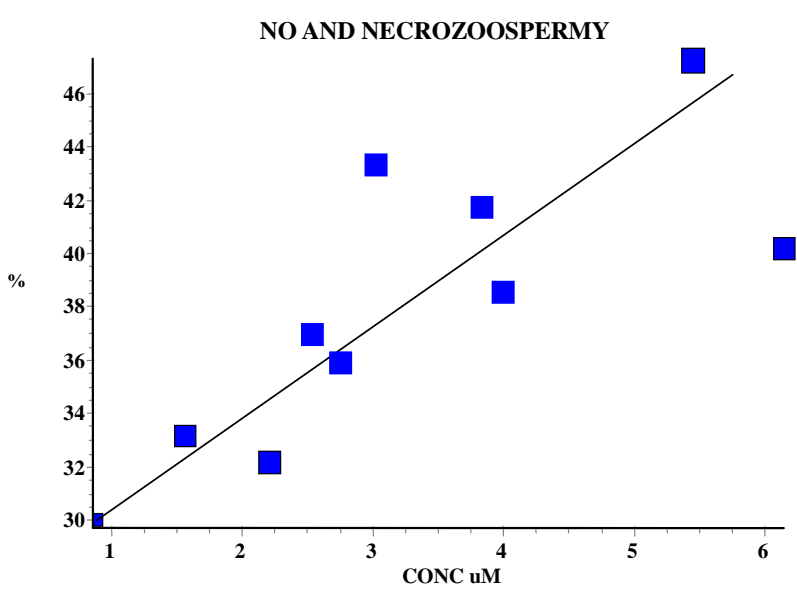

Figure 5. Correlation between seminal NO levels and necrozoospermy before and after treatment

Before antibiotic treatment, the rate of apoptosis determined by TUNEL technique in the study population was $30.46 \%$. Range $11 \%$ - 90\%. There was no correlation between apoptosis parameters and necrozoospermy $(\mathrm{r}=$ $0.11, \mathrm{p}>0.05)$. There was no correlation between sperm cell apoptosis and NO levels $(\mathrm{r}=0.18, \mathrm{p}>0.05)$.

\section{Discussion}

Seminal tract infections are often related to fertility problems in men [11]. We have observed a direct correlation betwen NO levels and sperm concentration and inversely with sperm motility. This is because the main sources of NO in the semen are the same gamete, many of those with excess residual cytoplasm and not seminal leukocytes. This explains why there is an increase in NO levels in seminal plasma samples with low white blood cells count.

$\mathrm{NO}$ is a free radical having an unpaired electron, which in low concentrations is required for sperm flagellar motility. At high concentrations is detrimental to the vitality of the gametes $[12,13]$.

Among the antibiotics that penetrate the prostatic half, 
fluoroquinolones can achieve a concentration several times greater than plasma so formed themselves into a group of proven effectiveness. Bundrick et al. reported that the concentration of ciprofloxacin and levofloxacin in the prostatic fluid was 0.18 and $1.42 \mathrm{mg} / \mathrm{dl}$ respectively. An important fact is that levofloxacin incorporated into its spectrum antimicrobial effective action against $U$. urealyticum and C. trachomatis.

In the present study we observed that antibiotic therapy with levofloxacin improved semen parameters, such as count motility and necrozoospermy in infertile men with sperm tract infection and NO levels are related to count, motility and necrozoospermia parameters but not with the percentage of sperm with DNA fragmentation.

In our study population we have not used antiinflammatory drugs or additives such as antioxidants, previous studies have shown alter semen parameters in patients with inflammation of the spermatic way $[14,15]$.

In conclusion treatment with levofloxacin for, at least 28 days improves seminal parameters in infertile patients with spermatic tract infection. Doses of $500 \mathrm{mg}$ for 56 days had better bacteriological cure rate over the 28 day regimen.

At difference of observed previously in patients with varicocele [16], here we observed a significant correlation between nitric oxide concentrations and semen profile. Probably the cause is that the infammatory process in seminal tract infections is higher than in varicocele and the physiopathology of testicular damage in both entities is different.

Nitric oxide but not TUNEL had good correlation with necrozoospermy parameters in this population.

\section{REFERENCES}

[1] A. J. Schaeffer, S. C. Wu, A. M. Tennenberg and J. B. Kahn, "Treatment of Chronic Bacterial Prostatitis with Levofloxacin and Ciprofloxacin Lowers Serum Prostate Specific Antigen," Journal of Urology, Vol. 174, No. 1, 2005, pp. 161-164. doi:10.1097/01.ju.0000162017.24965.2b

[2] W. Bundrick, S. P. Heron, P. Ray, W. M. Schiff, A. M. Tennenberg, et al., "Levofloxacin Versus Ciprofloxacin in the Treatment of Chronic Bacterial Prostatitis: A Randomized Double-Blind Multicenter Study," Journal of Urology, Vol. 62, No. 3, 2006, pp. 537-541. doi:10.1016/S0090-4295(03)00565-X

[3] L. Liu, Q. Li, P. Han, X. Li, H. Zeng, Y. Zhu and Q. Wei, "Evaluation of Interleukin-8 in Expressed Prostatic Secretion as a Reliable Biomarker of Inflamation in Benign Prostatic Hyperplasia," Urología, Vol. 191, 2009, pp. 7377.

[4] S. Allameni, C. Naughton, R. Sharma, A. Thomas and A. Agarwal, "Increased Seminal Reactive Oxigen Species Levels in Patients with Varicoceles Correlate with Varicocele Grade but Not with Testis Size," Fertility and Ste- rility, Vol. 82, No. 6, 2004, pp. 1684-1686. doi:10.1016/j.fertnstert.2004.04.071

[5] M. Benchaib, V. Braun, J. Lornage, S. Hadj, B. Salle, et al., "Sperm DNA Fragmentation Decreases the Pregnancy Rate in an Assisted Reproductive Technique," Human Reproduction, Vol. 18, No. 5, 2003, pp. 1023-1028. doi:10.1093/humrep/deg228

[6] World Health Organization, "WHO Laboratory Manual for the Examination of Human Semen and Sperm-Cervical Mucus Interaction," 4th Edition, Cambridge University Press, Cambridge, 1999.

[7] T. F. Kruger, R. Menkveld and F. S. H. Stander, "Sperm Morphologic Features as a Prognostic Factor in in Vitro Fertilization," Fertility and Sterility, Vol. 46, No. 6, 1986, pp. 1118-1123.

[8] L. Gandini, F. Lombardo, D. Paoli, L. Caponecchia, G. Familiari, et al., "Study of apoptotic DNA fragmentation in human spermatozoa," Human Reproduction, Vol. 15, No. 4, 2000, pp. 830-839. doi:10.1093/humrep/15.4.830

[9] J. Tesarik, F. Ubaldi, L. Rienzi, F. Martinez, M. Iacobelli, et al., "Caspase-Dependent and -Independent Dna Fragmentation in Sertoli and Germ Cells from Men with Primary Testicular Failure: Relationship with Histological Diagnosis," Human Reproduction, Vol. 19, No. 2, 2004, pp. 254-261. doi:10.1093/humrep/deh081

[10] W. R. Tracey, J. Tse and G. Cater, "LipopolysaccharideInduced Changes in Plasma Nitrite and Nitrate Concentrations in Rat and Mice: Pharmacological Evaluation of Nitric Oxide Syntase Inhibitors," Journal of Pharmacology and Experimental Therapeutics, Vol. 272, No. 3, 1995, 1011-1015.

[11] E. Moretti, S. Capitani, N. Figura, A. Pomolli, M. G. Federico, et al., "The Presence of Bacteria Species in Semen and Sperm Quality," Journal of Assisted Reproduction and Genetics, Vol. 26, No. 1, 2009, pp. 47-56. doi:10.1007/s10815-008-9283-5

[12] F. Mazzilli, T. Rossi, M. Marchesini, C. Ronconi and F. Dondero, "Superoxide Anion in Human Semen Related to Seminal Parameters and Clinical Aspects," Fertility and Sterility, Vol. 62, No. 4, 1994, pp. 862-868.

[13] N. P. Lee and C. Y. Cheng, "Nitric Oxide and Cyclic Nucleotides: Their Roles in Junction Dynamics and Spermatogenesis," Advances in Experimental Medicine and Biology, Vol. 636, No. 1, 2008, pp. 172-185.

[14] E. Vicari, et al., "Antioxidant Treatment with Carnitines Is Effective in Infertile Patients with Prostatovesiculoepididymitis and Elevated Seminal Leukocyte Concentrations after Treatment with Nonsteroidal Antiinflammatory Compounds," Fertility and Sterility, Vol. 78, No. 6, 2002, pp. 1203-1208.

[15] J. E. Lackner, R. Herwig, J. Schmidbauer, G. Schatzl, C. Kratzik, et al., "Correlation of Leukocytospermia with Clinical Infection and the Positive Effect of Antiinflammatory Treatment on Semen Quality," Fertility and Sterility, Vol. 86, No. 3, 2006, pp. 601-605. doi:10.1016/j.fertnstert.2006.01.032

[16] P. M. Rodriguez, et al., "Predictors of Improved Seminal Parameters and Fertility after Varicocele Repair in Young Adults," Andrologia, Vol. 41, No. 5, 2009, pp. 277-281. 